\title{
Serological cancer-associated protein biomarker levels at bowel endoscopy: Increased risk of subsequent primary malignancy
}

\author{
Thomas B. Piper $^{\mathrm{a}, *}$, Hans J. Nielsen ${ }^{\mathrm{a}, \mathrm{b}, 1}$ and Ib Jarle Christensen ${ }^{\mathrm{a}}$ \\ ${ }^{a}$ Department of Surgical Gastroenterology, Hvidovre Hospital, Hvidovre, Denmark \\ ${ }^{\mathrm{b}}$ Institute of Clinical Medicine, University of Copenhagen, Copenhagen, Denmark
}

Received 10 January 2021

Accepted 21 December 2021

\begin{abstract}
.
BACKGROUND: It was previously shown in three subpopulations that subjects not identified with colorectal cancer (CRC) at bowel endoscopy, but with increased serological cancer-associated protein biomarker levels had an increased risk of being diagnosed with subsequent malignant diseases. Objective: The aim of the present study was to perform a pooled analysis of subjects from the three subpopulations and subsequently validate the results in an independent study. The study population denoted the training set includes $\mathrm{N}=4,076$ subjects with symptoms attributable to CRC and the independent validation set $\mathrm{N}=3,774$ similar subjects.

METHODS: Levels of CEA, CA19-9, TIMP-1 and YKL-40 were determined in blood samples collected prior to diagnostic bowel endoscopy. Follow-up of subjects not diagnosed with CRC at endoscopy, was ten years and identified subjects diagnosed with primary intra- or extra-colonic malignant diseases. The primary analysis was time to a newly diagnosed malignant disease and was analyzed with death as a competing risk in the training set. Subjects with HNPCC or FAP were excluded. The cumulated incidence was estimated for each biomarker and in a multivariate model. The resulting model was then validated on the second study population.

RESULTS: In the training set primary malignancies were identified in $515(12.6 \%)$ of the 4,076 subjects, who had a colorectal endoscopy with non-malignant findings. In detail, 33 subjects were subsequently diagnosed with CRC and 482 subjects with various extra-colonic cancers. Multivariate additive analysis of the dichotomized biomarkers demonstrated that CEA $(\mathrm{HR}=1.50,95 \% \mathrm{CI}: 1.21-1.86, p<0.001)$, CA19-9 $(\mathrm{HR}=1.41,95 \% \mathrm{CI}: 1.10-1.81, p=0.007)$ and TIMP-1 (HR $=1.25$ 95\% CI: $1.01-1.54, p=0.041)$ were significant predictors of subsequent malignancy. The cumulated incidence at 5 years landmark time was $17 \%$ for those subjects with elevated CEA, CA19-9 and TIMP-1 versus $6.7 \%$ for those with low levels of all. When the model was applied to the validation set the cumulated 5-year incidence was $10.5 \%$ for subjects with elevated CEA, CA19-9 and TIMP-1 and 5.6\% for subjects with low levels of all biomarkers. Further analysis demonstrated a significant interaction between TIMP-1 and age in the training set. The age dependency of TIMP-1 indicated a greater risk of malignancy in younger subjects if the biomarker was elevated. This observation was validated in the second set.

CONCLUSION: Elevated cancer-associated protein biomarker levels in subjects with non-malignant findings at large bowel endoscopy identifies subjects at increased risk of being diagnosed with subsequent primary malignancy. CEA, CA19-9 and TIMP-1 were significant predictors of malignant disease in this analysis. TIMP-1 was found dependent on age. The results were validated in an independent symptomatic population.
\end{abstract}

Keywords: Colorectal cancer, colorectal neoplasia, malignancy, protein biomarkers, endoscopy

\footnotetext{
*Corresponding author: Thomas B. Piper, MD, Department of Surgical Gastroenterology 360, Hvidovre Hospital, University of Copenhagen, DK-2650 Hvidovre, Denmark. Tel.: +45 38622436; Fax: +45 38623760; E-mail: thomas.baastrup. piper@regionh.dk.

${ }^{1}$ Deceased during the writing process.
} 


\section{Introduction}

Globally, the incidence of malignant diseases is increasing, due to an increasing elderly population, environmental factors, altered lifestyles and due to improved diagnostic achievements. In total, cancer incidences rose from 14.1 million in 2012 to 17.5 million in 2015. The registered number of cancer related deaths rose from 8.2 million to 8.7 million in the same period [1,2]. Global cancer incidence was estimated to rise to 18.1 million in 2018 with 9.6 million cancer related deaths $[1,3]$.

Ranked, the 5 most common causes of cancer deaths are lung-, gastric-, liver-, breast- and colorectal cancer [1]. Colorectal cancer (CRC) is diagnosed in 1.85 million people every year and 550,000 die from the disease worldwide [1]. The median age at diagnosis is approximately 70 years $[4,5]$. Treatment of CRC and other cancers is costly, and both morbidity and mortality increase with the stage of cancer at time of diagnosis and total annual economic costs of cancer in 2010 was estimated to approximately US\$ 1.16 trillion [6].

Early detection of CRC or removal of potentially premalignant adenomas are of the utmost importance to prevent morbidity and mortality caused by CRC [5].

Early detection of other cancers may improve the patient disease course and prognosis, but it is important to note that overdiagnosis and harm by screening should be taken into account and that cost-benefit analyses before screening are important [7].

Previously our research group has analyzed three different subgroups from a population recruited in 2004-05 including 4,990 subjects with symptoms attributable to CRC in prospective studies. The three studies included subjects without findings at colorectal endoscopy, subjects with non-neoplastic lesions at colorectal endoscopy and subjects with colorectal adenomas [8-10]. Results from these three studies demonstrated an association between elevated cancer-associated biomarker levels and development of subsequent malignant diseases including CRC [8-10].

Levels of the biomarker TIMP-1 has previously been described as dependent on age in some studies $[11,12]$, but to our knowledge, the possible age dependency has not been evaluated in a large cohort study.

The aim of the present study was to reevaluate whether subjects without malignant findings at bowel endoscopy, but with elevated serological cancer-associated protein biomarker levels have an increased risk of being diagnosed with subsequent malignant diseases. We performed a pooled analysis of the results on the cancer-associated biomarkers already published in the three mentioned studies $(\mathrm{N}=4,076)$. The biomarkers were carcinoembryonic antigen (CEA), plasma tissue inhibitor of metalloproteinases-1 (TIMP-1), cancer antigen 19-9 (CA19-9) and human cartilage glycoprotein-39 (YKL-40). The results were then validated in an independent symptomatic population referred to colonoscopy $(\mathrm{N}=3,774)$. Furthermore, an aim of the present study was to test a possible age dependency of TIMP-1 in a large population and to validate the result in a comparable, independent symptomatic population.

Four serological cancer-associated protein biomarkers were analyzed: CEA, CA19-9, TIMP-1 and YKL-40. The four biomarkers were chosen before inclusion of subjects in 2004-2005 because they showed promising results in previous studies on early detection of colorectal neoplasia [13-17] - the results which have been tested in various studies subsequently [18-24].

Both biomarkers, CEA and CA19-9, have been related to CRC for decades. CEA was the first soluble biomarker used for CRC surveillance and is still being used for monitoring CRC. CEA is significantly associated with the presence of CRC and is strongly dependent on CRC stage [5]. CA19-9 is commonly used as a biomarker for diagnosis and surveillance of pancreatic cancer, but has also been associated with breast cancer, ovarian cancer, cancer in the stomach and esophagus, with hepatocellular carcinoma and CRC [25-30]. Elevated concentrations of TIMP-1 are associated to various malignancies such as non-small-cell lung cancer, renal cancer, ovarian cancer and CRC [5, 31-33]. YKL-40 is secreted 
by and associated with different types of solid tumors, including several types of adenocarcinomas, glioblastoma, melanoma and small cell lung carcinoma [34].

The present study was performed to validate former results - that elevated cancer-associated biomarkers identify subjects at increased risk of subsequent malignancy and to test the age dependency of TIMP-1.

Blood sample data from two such large and comparable study populations are limited. The results of the present study may aid to develop a blood screening platform, including the analyzed biomarkers and other promising biomarkers. Attempts of producing a screening platform for occult cancer has been done [35, 36], but to our knowledge no reasonably priced platform, able to distinguish between different malignancies, is currently being used. The combination of different blood biomarkers with cancer type specificity and a high combined sensitivity could be promising for such a platform. In time, frequent out-patient follow-up may detect early neoplastic lesions. Subsequently, patients may be referred to the right treatment earlier and both patients and society may benefit from this.

\section{Methods}

The 4,990 subjects in the training set were included in a prospective, population-based multicenter study conducted in 2004/05 at six collaborating hospitals in Denmark. The subjects had symptoms attributable to CRC or were included in the Danish HNPCC surveillance program. According to a validated standard operational procedure blood samples were collected prior to endoscopy, handled into serum, EDTA plasma and buffy-coats and frozen at $-80^{\circ} \mathrm{C}$ within two hours. The frozen samples were under 24/7 electronic surveillance. Plasma levels of the prespecified protein biomarkers CEA, CA19-9, TIMP-1 and serum levels of YKL-40 were determined as shown previously to identify a potential relation to endoscopy findings [8-10].

Subjects were stratified into five ranked groups at time of inclusion depending on the primary endoscopy findings: 1: CRC; 2: Extra-colonic cancer; 3: Colorectal adenomas; 4: Non-neoplastic findings, mainly diverticula, and 5: Clean colorectum (Fig. 1). In the present study, only subjects without malignancy at time of inclusion, were included. Hence, subjects from the three initial ranked groups with colorectal adenomas, non-neoplastic findings and clean colorectum. The study was approved by the Ethics Committee of the Capital Region of Denmark (KF01-080/03), which represents all collaborating hospitals, and in addition approved by the Danish Data Protection Agency (2003-41-3312). Before inclusion, all subjects signed an informed consent form. Symptoms or adherence to a HNPCC surveillance program guided whether a subject underwent sigmoidoscopy or colonoscopy. Exclusion criteria were less than 18 years of age, a previous diagnosis of CRC or inability to comply with the protocol [5]. Subjects with findings of adenomas at primary sigmoidoscopy have been offered subsequent colonoscopy, which was not uniformly performed due to patient refusal. Subjects, who underwent primary sigmoidoscopy without findings of colorectal pathology, but with persisting symptoms were offered an additional colonoscopy. Subjects, who had been examined with primary colonoscopy or examined with primary sigmoidoscopy and subsequent colonoscopy but had persisting symptoms were offered additional examinations with ultra-sonography, computerized axial tomography or magnetic resonance imaging.

The inclusion of all subjects was finalized by December $31^{\text {st }}, 2005$. Audits were performed both onsite and electronically via patient files and existing databases. After termination of the study inclusion, levels of the 4 protein biomarkers were analyzed using validated analysis platforms. CEA, CA19-9 and TIMP-1 were determined in EDTA plasma using the Abbott ARCHITECT ${ }^{\circledR}$ i2000 automated immunoassay system [5]. Levels of the cancer-associated biomarker YKL-40 were determined in serum by a commercial ELISA platform [18] with an on-site detection limit $10 \mathrm{ng} / \mathrm{mL}$, intra-assay coefficients of variability (CV) of $5 \%$ and inter-assay $\mathrm{CV}$ of $<6 \%$. 

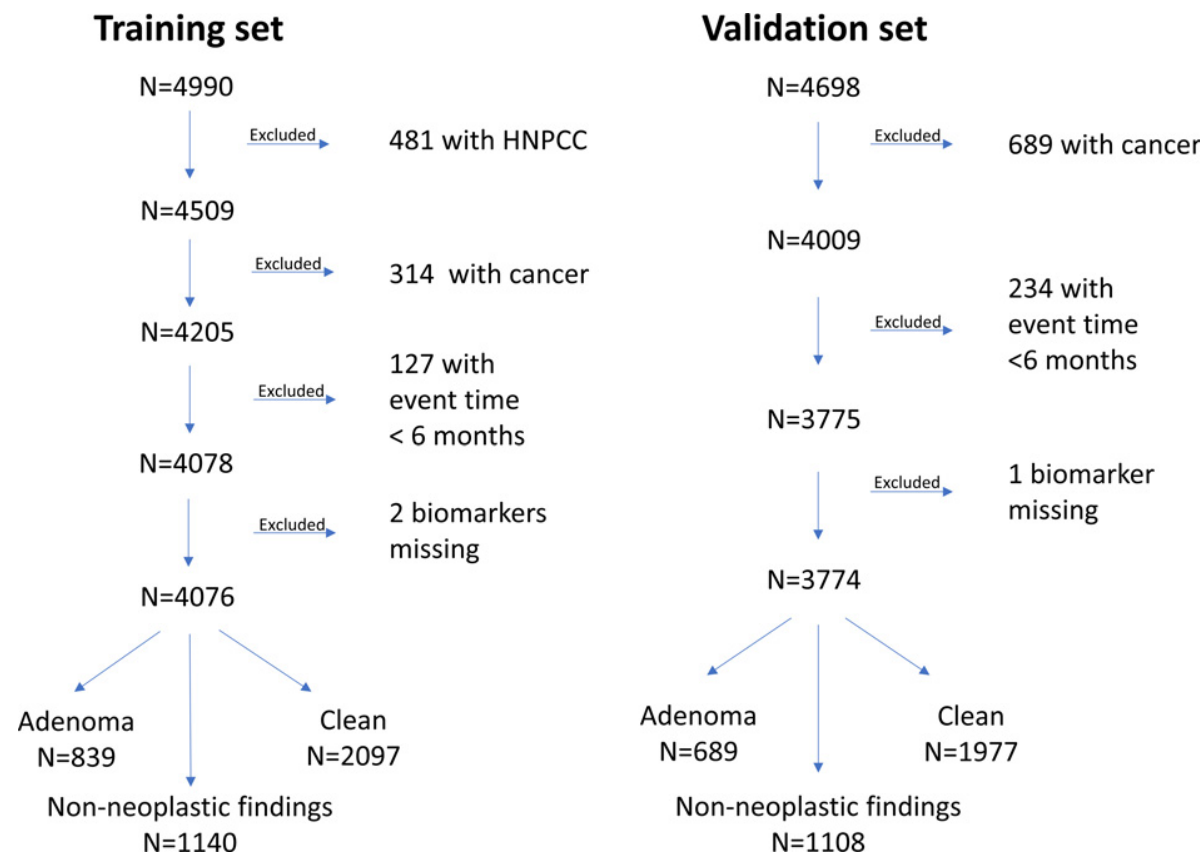

Fig. 1. Flow chart showing the excluding process of subjects from the training and validation sets in the present study, ending up with the two study populations: Training set $\mathrm{N}=4,076$ and validation set $\mathrm{N}=3,774$. The training set was previously subdivided into an adenoma subgroup, a subgroup with non-neoplastic findings and a subgroup with clean colorectum. The same is shown for the validation set.

All findings at endoscopy were recorded in a database using the International Classification of Diseases-10 (ICD-10) system. In addition, gender and age were recorded for all subjects. Adenomas found at endoscopy were stratified by the current classification as either at high- or low-risk. Subjects were followed prospectively from primary endoscopy in 2004/05 and primary malignancies were registered. Corresponding to a follow up period of 7 to 10 years depending on the time of the audits. Skin cancers - except malignant melanomas and ten subjects with spinocellular carcinomas either with local invasion or disseminated cancer - were not included. Events registered within 6 months from the initial bowel endoscopy were not included for further analysis $(\mathrm{N}=127)$. Subjects with HNPCC or FAP were excluded, as described previously [10].

The validation set included 4,698 independent symptomatic subjects referred to first time ever colonoscopy from May 1, 2010 to November 30, 2012. Subjects had blood samples collected before diagnostic colonoscopy. The blood samples were handled according to a validated standard operational procedure, exactly as the samples from the training set as described. Protein biomarkers, CEA, CA19-9 and TIMP-1, were analyzed using the same assays as in the training set, whereas YKL-40 was not determined in the validation set. Subjects were stratified as in the training set. Non-malignant findings were identified in 4,009 subjects analyzed in the present study (Fig. 1). Events registered within 6 months from the initial bowel endoscopy were not included for further analysis $(\mathrm{N}=234)$. Subjects with malignant findings and high-risk adenomas have been described earlier [37].

\section{Statistics}

The individuals included were those without malignant finding at endoscopy, and without a cancer diagnosis within 6 months from that date. The time from this landmark to any cancer (except ordi- 
nary skin cancer) were recorded, if no event occurred then the individual was censored. The study comprises a training dataset based on prospectively accrued individuals with symptoms attributable to $\mathrm{CRC}$ and a validation set collected from an independent study. The principle analysis was based on the Cox proportional hazards model with death as a competing risk [38]. Four biomarkers were assessed, CEA, TIMP-1, CA19-9 and YKL-40. The markers were scored as the log of the value (base 2) or as elevated/normal. The latter is the $90^{\text {th }}$ percentile of the log transformed marker level regressed on age using values from individuals referred to colonoscopy due to symptoms but without any finding, comorbidity or use of prescription drugs. This procedure resulted in an age corrected elevated/normal binary variable. Univariate as well as multivariate analyses were performed, the latter including biomarkers as well as age and gender. Results were present as hazard ratios (HR) with 95\% confidence limits and as the incidence rates. For the models using markers on the continuous scale, the first quartile was defined as low and the third quartile as high calculating the cumulative incidence entering these values in the regression equation based on the continuous model specification. The training set was used for identification of relevant signature for prediction of subsequent cancer, and this was in turn applied to the validation set. The initial model was selected from the training set including the significant covariates in the multivariate setting. Analyses included tests for relevant interactions in particular between markers and age as well as the status (clean colon, non-malignant finding or adenoma) at the examination by including the interaction term in the regression model. Final models were stepwise reduced although always including age and gender, noting that age was a confounding variable. The final model was then locked and applied to the validation set.

The level of significance was set to $5 \%$. Calculations were done using SAS v9.4.

\section{Results}

In total, 4,076 subjects from the training set were included in the present study; 2,242 (55\%) were women. The validation set included 3,774 subjects; 2041 (54\%) were women. The median ages in the two sets were 60.9 years (18.5-97.3) and 63.1 years (18.1-96.0), respectively. Symptoms from the subjects in the training set recorded at inclusion were distributed as follows: 1,875 (46\%) had pain, 1,465 (36\%) had bleeding, 1,835 (45\%) had altered bowel habits, $687(17 \%)$ had weight loss, $33(1 \%)$ had unspecific symptoms and finally $396(10 \%)$ subjects could not inform about any symptoms, but were referred by a physician because of symptoms attributable to CRC, with some subjects having several symptoms. In the validation set, 1,818 (48\%) had pain, 1,341 (36\%) had bleeding, 2,371 (63\%) had altered bowel habits, 903 (24\%) had weight loss, 498 (13\%) had anemia, 135 (4\%) palpable tumors, $796(21 \%)$ had meteorism and $105(3 \%)$ had no reported symptoms. The median marker levels in the training study were the following: YKL-40: $69 \mathrm{ng} / \mathrm{ml}$ (6-3502), CEA $1.8 \mathrm{ng} / \mathrm{ml}$ (0-738), CA19-9 $5.5 \mathrm{ng} / \mathrm{ml}$ (2-23480) and TIMP-1 $86 \mathrm{ng} / \mathrm{ml}$ (27-818). In the validation study, the median levels were $2.1 \mathrm{ng} / \mathrm{ml}$ (0-37) for CEA, $8.1 \mathrm{ng} / \mathrm{ml}$ (2.0-4338) for CA19-9 and $98 \mathrm{ng} / \mathrm{ml}$ (30-1064) for TIMP-1.

Primary malignancies were identified in 515 (12.6\%) of the 4,076 subjects with non-malignant findings at the primary bowel endoscopy in the training set (Table 1). In detail, 33 subjects were subsequently diagnosed with CRC and 482 subjects with various extra-colonic cancers. In total, 455 subjects had high risk adenomas and 384 subjects had low risk adenomas - defined in a previous study [10].

The rate of primary malignancy in the study period was highest in the group diagnosed with adenoma at bowel endoscopy (18\%) in contrast to those with non-malignant findings $(13 \%)$ and clean colon $(11 \%)$, however tests for interaction between the biomarkers and findings were not significant suggesting that the biomarkers had similar impact. $P$-values for the interaction terms for the continuous model specification were 0.58 (YKL-40), 0.83 (TIMP-1), 0.38 (CA19-9) and 0.78 (CEA). For the dichotomized models, the $p$-values were $0.18,0.55,0.84$ and 0.93 , respectively. 
Table 1

The number and location of cancers detected in training and validation studies

\begin{tabular}{|c|c|c|c|}
\hline \multirow[t]{2}{*}{ Cancer diagnosis } & & \multicolumn{2}{|c|}{ Study } \\
\hline & & Training & Validation \\
\hline \multirow[t]{2}{*}{ Nose, moth and throat } & Number (N) & 16 & 11 \\
\hline & $\%$ & 2.00 & 1.38 \\
\hline \multirow[t]{2}{*}{ Gastrointestinal tract } & $\mathrm{N}$ & 43 & 32 \\
\hline & $\%$ & 5.38 & 4.01 \\
\hline \multirow[t]{2}{*}{ Liver, gallbladder, pancreas } & $\mathrm{N}$ & 16 & 17 \\
\hline & $\%$ & 2.00 & 2.13 \\
\hline \multirow[t]{2}{*}{ Bronchi, lung and pleura } & $\mathrm{N}$ & 61 & 36 \\
\hline & $\%$ & 7.63 & 4.51 \\
\hline \multirow[t]{2}{*}{ Malignant melanoma } & $\mathrm{N}$ & 15 & 20 \\
\hline & $\%$ & 1.88 & 2.50 \\
\hline \multirow[t]{2}{*}{ Connective tissue } & $\mathrm{N}$ & 3 & 2 \\
\hline & $\%$ & 0.38 & 0.25 \\
\hline \multirow[t]{2}{*}{ Breast } & $\mathrm{N}$ & 56 & 36 \\
\hline & $\%$ & 7.01 & 4.51 \\
\hline \multirow[t]{2}{*}{ Female genitalia } & $\mathrm{N}$ & 49 & 9 \\
\hline & $\%$ & 6.13 & 1.13 \\
\hline \multirow[t]{2}{*}{ Male genitalia } & $\mathrm{N}$ & 176 & 54 \\
\hline & $\%$ & 22.03 & 6.76 \\
\hline \multirow[t]{2}{*}{ Kidney and urinary tract } & $\mathrm{N}$ & 25 & 27 \\
\hline & $\%$ & 3.13 & 3.38 \\
\hline \multirow[t]{2}{*}{ Nervous system } & $\mathrm{N}$ & 5 & 5 \\
\hline & $\%$ & 0.63 & 0.63 \\
\hline \multirow[t]{2}{*}{ Thyroidea } & $\mathrm{N}$ & 1 & 2 \\
\hline & $\%$ & 0.13 & 0.25 \\
\hline \multirow[t]{2}{*}{ Thorax } & $\mathrm{N}$ & 1 & . \\
\hline & $\%$ & 0.13 & . \\
\hline \multirow[t]{2}{*}{ Cancer not specified and metastasis } & $\mathrm{N}$ & 15 & 1 \\
\hline & $\%$ & 1.88 & 0.13 \\
\hline \multirow[t]{2}{*}{ Different types of leukemia } & $\mathrm{N}$ & 33 & 32 \\
\hline & $\%$ & 4.13 & 4.01 \\
\hline Total N & & 515 & 284 \\
\hline
\end{tabular}

The expected cancer incidence per year for the Danish population in 2005 was calculated based on the NORDCAN database [39], excluding non-melanoma skin cancer and was found to be $1.35 \%$ which is lower than the overall observed cancer incidence of $2.11 \%$ in the training dataset.

In the validation set, primary malignancies were identified in $284(7.5 \%)$ of the 3,774 subjects. The distribution of cancer diagnoses is listed in Table 1.

Univariate analyses made for the training set showed that all dichotomized biomarkers were significant for detecting malignancy; all had $p<0.001$. Univariate analyses of markers on a continuous $\log$ scale base 2 were also significant (Table 2).

Multivariate analyses of the markers on a log transformed scale showed that YKL-40 $(p=0.11)$ and TIMP-1 $(p=0.078)$ were not significant, whereas CEA $(\mathrm{HR}=1.1395 \% \mathrm{CI}: 1.04-1.23, p=0: 003)$ and CA19-9 $(\mathrm{HR}=1.1095 \% \mathrm{CI}: 1.03-1.17, p=0.004)$ were significant (Table 2). Multivariate additive analyses of the dichotomized markers demonstrated that CEA (HR=1.50, 95\% CI:1.21-1.86, 
Table 2

Univariate and multivariate analysis, including calculated Hazard Ratios, $p$-value's and confidence intervals, for YKL-40, TIMP-1, CA19-9 and CEA

\begin{tabular}{|c|c|c|c|c|c|c|c|c|c|c|c|c|c|c|c|c|}
\hline \multirow[t]{3}{*}{ Marker } & \multicolumn{16}{|c|}{ Model } \\
\hline & \multicolumn{4}{|c|}{ Univariate continuous } & \multicolumn{4}{|c|}{ Univariate binary } & \multicolumn{4}{|c|}{ Multivariate continuous } & \multicolumn{4}{|c|}{ Multivariate binary } \\
\hline & $\begin{array}{c}\text { Hazard } \\
\text { Ratio }\end{array}$ & $\begin{array}{c}95 \% \text { Lower } \\
\text { Confidence } \\
\text { Limit for } \\
\text { Hazard } \\
\text { Ratio } \\
\end{array}$ & $\begin{array}{c}95 \% \text { Upper } \\
\text { Confidence } \\
\text { Limit for } \\
\text { Hazard } \\
\text { Ratio } \\
\end{array}$ & $\begin{array}{c}\mathrm{Pr}> \\
\text { ChiSq }\end{array}$ & $\begin{array}{c}\text { Hazard } \\
\text { Ratio }\end{array}$ & $\begin{array}{c}95 \% \text { Lower } \\
\text { Confidence } \\
\text { Limit for } \\
\text { Hazard } \\
\text { Ratio } \\
\end{array}$ & $\begin{array}{c}95 \% \text { Upper } \\
\text { Confidence } \\
\text { Limit for } \\
\text { Hazard } \\
\text { Ratio } \\
\end{array}$ & $\begin{array}{c}\mathrm{Pr}> \\
\text { ChiSq }\end{array}$ & $\begin{array}{c}\text { Hazard } \\
\text { Ratio }\end{array}$ & $\begin{array}{c}95 \% \text { Lower } \\
\text { Confidence } \\
\text { Limit for } \\
\text { Hazard } \\
\text { Ratio } \\
\end{array}$ & $\begin{array}{c}95 \% \text { Upper } \\
\text { Confidence } \\
\text { Limit for } \\
\text { Hazard } \\
\text { Ratio } \\
\end{array}$ & $\begin{array}{c}\mathrm{Pr}> \\
\text { ChiSq }\end{array}$ & $\begin{array}{c}\text { Hazard } \\
\text { Ratio }\end{array}$ & $\begin{array}{c}95 \% \text { Lower } \\
\text { Confidence } \\
\text { Limit for } \\
\text { Hazard } \\
\text { Ration } \\
\end{array}$ & $\begin{array}{c}95 \% \text { Upper } \\
\text { Confidence } \\
\text { Limit for } \\
\text { Hazard } \\
\text { Ration } \\
\end{array}$ & $\begin{array}{c}\text { Pr }> \\
\text { ChiSq }\end{array}$ \\
\hline YKL-40 & 1.18 & 1.10 & 1.26 & $<0.001$ & 1.23 & 1.00 & 1.51 & 0.050 & 1.07 & 0.98 & 1.17 & 0.108 & 1.04 & 0.83 & 1.30 & 0.738 \\
\hline TIMP-1 & 1.53 & 1.32 & 1.78 & $<0.001$ & 1.37 & 1.12 & 1.66 & 0.002 & 1.20 & 0.98 & 1.48 & 0.078 & 1.25 & 1.01 & 1.54 & 0.041 \\
\hline CA19-9 & 1.16 & 1.09 & 1.24 & $<0.001$ & 1.57 & 1.23 & 2.01 & $<0.001$ & 1.10 & 1.03 & 1.17 & 0.004 & 1.41 & 1.10 & 1.81 & 0.007 \\
\hline CEA & 1.22 & 1.13 & 1.32 & $<0.001$ & 1.62 & 1.31 & 2.00 & $<0.001$ & 1.13 & 1.04 & 1.23 & 0.003 & 1.50 & 1.21 & 1.86 & $<0.001$ \\
\hline
\end{tabular}


A

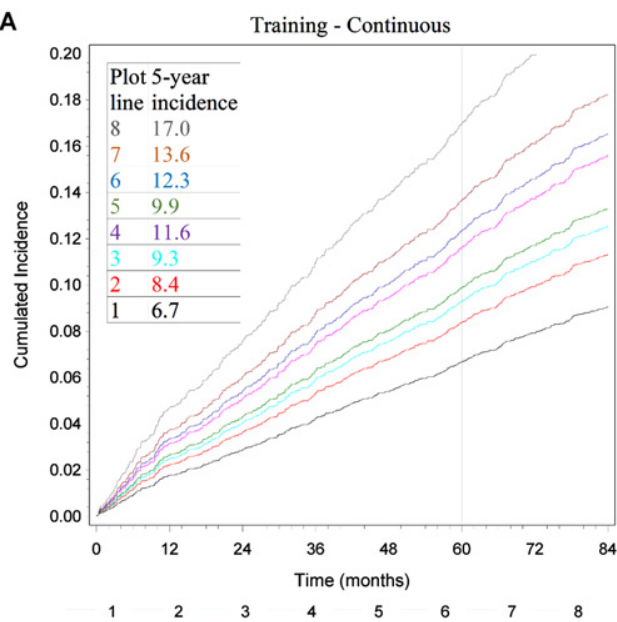

C

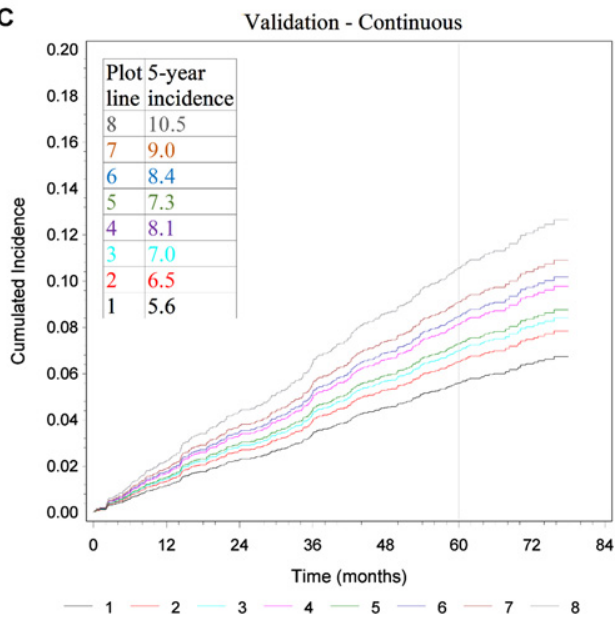

B

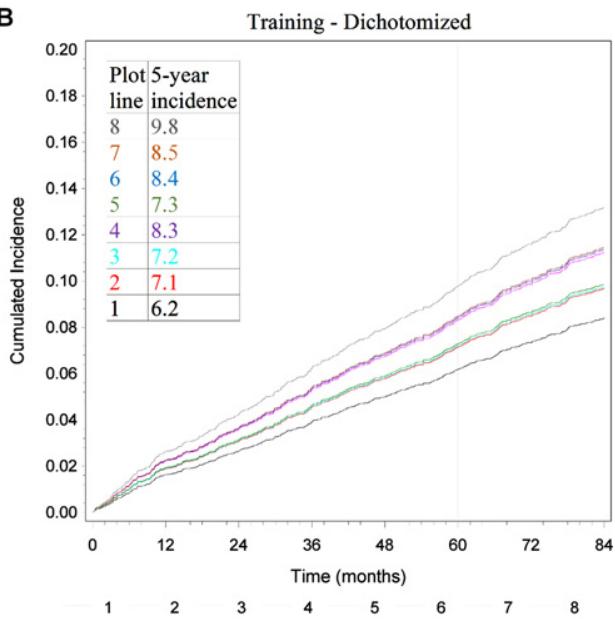

D

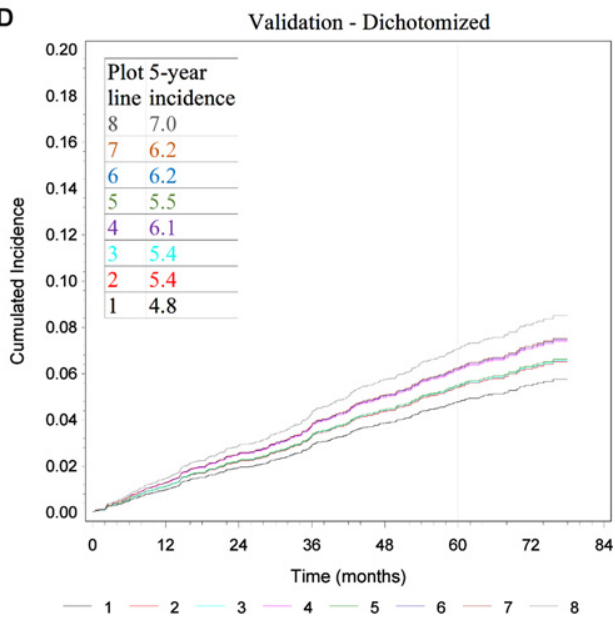

Fig. 2. Cumulated incidences for the additive multivariate models, $p<0.0001$. Plots A and B show results for the training set, plots $\mathrm{C}$ and $\mathrm{D}$ show results for the validation set. Plot $\mathrm{A}$ and $\mathrm{C}$ show continuous results, plots $\mathrm{B}$ and $\mathrm{D}$ show dichotomized results. The 5-year cumulated incidence of the eight plot lines is shown in A-D. For plots A-D: Line 1; CEA, CA19-9, TIMP-1 - all normal levels. Line 2; CEA and CA19-9 normal levels, TIMP-1 elevated level. Line 3; CEA and TIMP-1 normal levels, CA19-9 elevated level. Line 4; CEA normal level, TIMP-1 and CA19-9 elevated levels. Line 5; CA19-9 and TIMP-1 normal levels, CEA elevated level. Line 6; CA19-9 normal level, CEA and TIMP-1 elevated levels. Line 7; TIMP-1 normal level, CEA and CA19-9 elevated levels. Line 8; CEA, CA19-9, TIMP-1 - all elevated levels.

$p<0.001)$, CA19-9 $(\mathrm{HR}=1.41,95 \% \mathrm{CI}: 1.10-1.81, p=0.007)$ and TIMP-1 $(\mathrm{HR}=1.25$ 95\%CI: $1.01-1.54, p=0.041)$ were significant predictors of subsequent malignancy but YKL-40 $(p=0.74)$ was not (Table 2). When age and gender were added to these multivariate models CEA and CA19-9 remained significant, but TIMP-1 was not significant ( $p=0.26$ and $p=0.73$, respectively).

Figure 2A shows cumulated incidences for the significant continuous biomarkers CEA, CA19-9 and TIMP-1. The cumulated malignancy incidence at 5 years was $6.7 \%$ for those having normal levels of all 3 biomarkers increasing to $17.0 \%$ for those subjects with all biomarker levels elevated. A similar pattern was seen for markers evaluated on the dichotomized scale, for all marker levels at the first quartile, the cumulated incidence was $6.2 \%$ and $9.8 \%$ for those at the third quartile, Fig. $2 \mathrm{~B}$.

The results of applying the multivariate model using continuous levels to the validation set are shown in Fig. 2C. The cumulated incidence for subjects with normal biomarker levels was $5.6 \%$ in contrast to $10.5 \%$ for those with all three biomarker levels elevated. For the models entering markers on the 
Table 3

The results of multivariate analysis of the biomarkers CEA and CA19-9, including an interaction between TIMP-1 and age

\begin{tabular}{lccccccc}
\hline & \multicolumn{3}{c}{ Continuous model } & & \multicolumn{3}{c}{ Binary model } \\
\cline { 2 - 4 } & Hazard Ratio & $95 \%$ CI & $p$-value & & Hazard Ratio & $95 \%$ CI & $p$-value \\
\hline CEA & 1.11 & $1.02-1.20$ & 0.022 & & 1.57 & $1.26-1.94$ & $<0.0001$ \\
CA19-9 & 1.11 & $1.04-1.19$ & 0.0015 & & 1.48 & $1.15-1.91$ & 0.002 \\
TIMP-1 Age $=50$ & 1.72 & $1.31-2.27$ & & 1.47 & $1.09-1.98$ & $0.96-1.50$ \\
TIMP-1 Age $=60$ & 1.21 & $0.99-1.49$ & $<0.0001$ & & 1.20 & 0.0008 \\
TIMP-1 Age $=70$ & 0.86 & $0.70-1.05$ & & & 0.98 & $0.80-1.20$ & \\
\hline
\end{tabular}

For additive biomarkers, hazard ratios with $p$-values are presented and for the interaction between TIMP-1 and age, the $p$-values are shown with the hazard ratios for three different, randomly chosen but representative ages: 50, 60 and 70 years of age with confidence intervals (CI) presented in addition. 'TIMP-1 age $=50$, age $=60$ or age $=70$ ' represents the calculated hazard ratios of TIMP-1 of a 50-, 60- or 70-year-old subject.

dichotomized level, the cumulated incidence was $4.8 \%$ for those at the first quartile and $7.0 \%$ for those at the third quartile, Fig. 2D.

Multivariate analyses of CEA and CA19-9 levels, including an interaction between TIMP-1 and age, are shown in Table 3. Hazard ratios with $p$-values are presented for the additive marker effects. Additionally, hazard ratios with $p$-values for the interaction between TIMP-1 and age with three chosen and representative ages, 50, 60 and 70 years, are presented.

The importance of TIMP-1 decreased with age. For 70-year-old the difference was minimal whereas for 50-year-old, the hazard ratio was 1.67 in the continuous model and 1.44 in the dichotomized model. The cumulated incidences of malignancy are shown in Fig. 3 (A-D). Figure 3A show results of the continuous analyses for the training set; for 50-year-old the cumulated incidence was $4.1 \%$ if the levels of CEA, CA19-9 and TIMP-1, were normal increasing to 6.0\% for those with elevated TIMP1. The cumulated incidence for 60 -year-old was $6.0 \%$ increasing to $7.1 \%$, whereas for 70 -year-old the cumulated incidence was $8.7 \%$ vs $8.5 \%$, respectively. The corresponding cumulated incidences for the model with markers from the training set on a dichotomized scale for age 50 with normal or elevated TIMP-1 were : $3.9 \%$ and $5.2 \%$, for age $60: 6.1 \%$ and $6.7 \%$, and for age $70: 9.4 \%$ and $8.7 \%$, respectively (Fig. 3B). This shows a higher impact for TIMP-1 at lower age compared to higher age. The same calculations for the validation set is illustrated in Fig. 3C-D. Figure 3C shows the continuous results, and Fig. 3D the dichotomized results.

\section{Discussion}

The present study evaluated data from two large and comparable populations (training and validation sets), both with subjects having endoscopy done due to symptoms attributable to CRC. The overall results of the training set showed that increased levels of the cancer-associated biomarkers, CEA, CA19-9 and TIMP-1, were significant in detecting subjects at risk of developing a subsequent primary malignancy. All results were assessed in the validation set using the models developed in the training set.

The data on the training set have been published [8-10], and in these studies, new malignancies were included from the day of the inclusion. New data audits done on the training set in connection with the present study showed that some minor registration alterations were made previously due to staggered registration. Thus, some subjects with a newly detected extra colonic malignancy within months of the initial endoscopy likely had their neoplasia at time of endoscopy. To exclude subjects who might had 
A

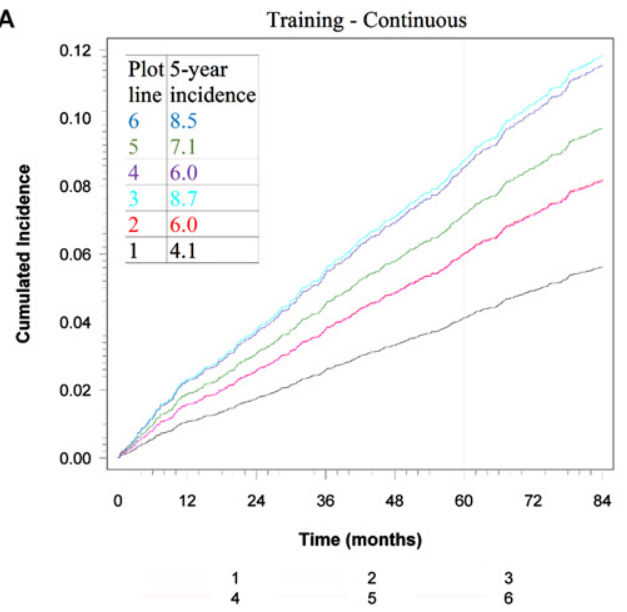

C

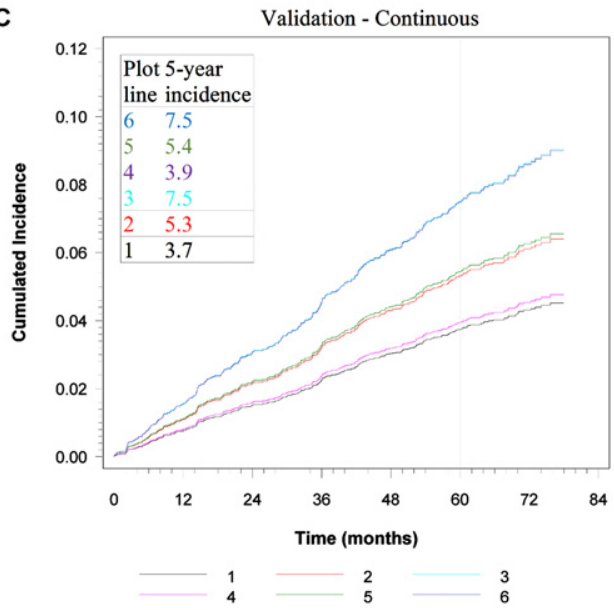

B

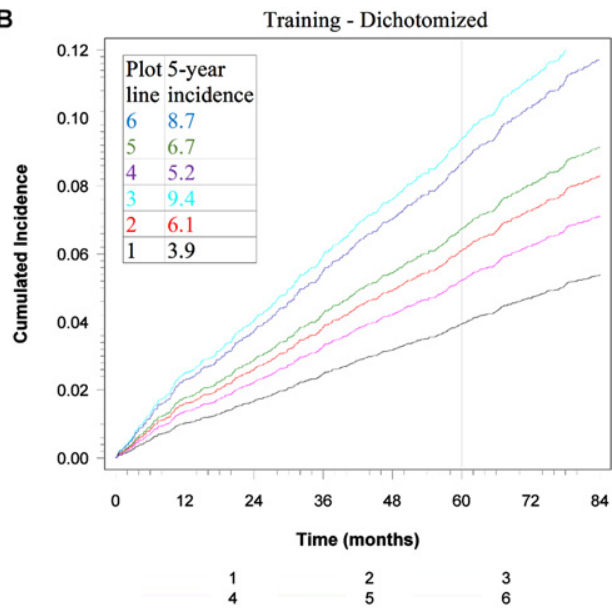

D

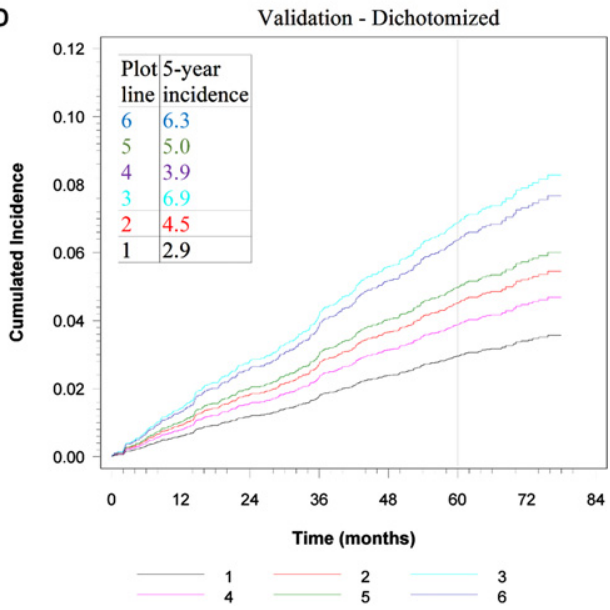

Fig. 3. Shows cumulated incidences for the model with interaction between TIMP-1 and age, $p<0.0001$. CEA and CA19-9 biomarker levels are normal. Plots $\mathrm{A}$ and $\mathrm{B}$ show results for the training set, Plots $\mathrm{C}$ and $\mathrm{D}$ show results for the validation set. Plot $\mathrm{A}$ and $\mathrm{C}$ show continuous results, plots $\mathrm{B}$ and $\mathrm{D}$ show dichotomized results. For plots A-D: Line 1 shows age 50 and normal TIMP-1. Line 2 shows age 60 and normal TIMP-1. Line 3 shows age 70 and normal TIMP-1. Line 4 shows age 50 and elevated TIMP-1. Line 5 shows age 60 and elevated TIMP-1. Line 6 shows age 70 and elevated TIMP-1.

an extra-colonic malignancy at inclusion a cut-off at six months after inclusion / primary endoscopy was chosen for the training set as well as the validation set. The cut-off was chosen assuming that symptom-giving extra-colonic malignancies would be diagnosed within six months of the endoscopy, as subjects were offered further examinations of malignancy if they had continuous symptoms and an endoscopic examination without an explanation of their symptoms.

Within the observation period of five years, $12.9 \%$ vs $7.5 \%$ of subjects in the training and validation sets, respectively, subsequently developed malignancy. The one-year malignancy incidence for the Danish general population was 1.35\% [39] which was lower than the overall one-year cancer incidence of $2.11 \%$ observed in the symptomatic training set. It was not surprising, that a population symptomatic of malignancy has a higher one-year incidence of cancer, than the general population. A part of the difference in cancer incidence between the two study populations can be explained by the registered prostate cancers. In the validation set 52 subjects were registered with prostate cancer vs 175 subjects in the training set. Different factors may have influenced on the difference in incidence of prostate cancer 
in the two populations. The training population was included between the years 2004 and 2005 and the validation population between 2010 and 2012. In Denmark the incidence rate of prostate cancer fell from 170 to 145 per 100.000 men between the years 2009 and 2010. The decrease was primarily due to the changed guidelines by the Danish Urologist Society in 2010, so that only genetically predisposed or symptomatic subjects should be screened with PSA. The high rate of prostate cancer in the training study was due to early detection by PSA-screening in the years before 2009 [40-42].

Thirty-three subjects from the training set developed CRC. Of those, 19 subjects developed a rightsided colon cancer. Those right-sided cancers may not have been detected because of an insufficient choice of primary endoscopy due to the lack of symptoms or as a result of missed premalignant lesions, as previously addressed $[9,10]$. Some subjects from the training set only had a proctoscopy or a sigmoidoscopy as primary endoscopy. In the validation set two subjects developed appendiceal cancer, but no subjects developed a colon or rectum cancer. All subjects in the validation set had a colonoscopy at inclusion, which may explain that no subjects developed CRC, because major precancerous lesions had been detected and resected at the time of inclusion.

Subjects in both populations developed various malignancies - almost the entire spectrum of the ICD10 classification. Yet the pattern was similar, and cancer types with large incidence and prevalence, such as prostate, lung, breast and colorectal cancers were represented as anticipated (Table 1).

Four cancer-associated biomarkers (CEA, CA19-9, TIMP-1 and YKL-40) were analyzed in the training set. Univariate analyses showed that all four biomarkers in the training set were significant predictors of primary malignancies $(p<0.001)$.

Both binary multivariate additive analyses and continuous multivariate additive analyses showed that CEA, CA19-9, female gender and age were significant, independent covariates. The fact that age was shown to be a significant independent covariate was not surprising as age is an important risk factor of developing a malignancy $[43,44]$.

Incidence curves were presented for the multivariate additive binary calculations of CEA, CA19-9 and TIMP-1 in the training set. The cumulated 5-year incidence was increased if one of the biomarkers was elevated compared to normal levels and increased even further to a substantial $17.0 \%$ cumulated 5-year incidence of subsequent malignancy if all biomarkers were elevated.

The multivariate analyses in the training set showed that YKL-40 was not a significant, independent biomarker, which made it possible to validate the other three biomarkers (CEA, CA19-9 and TIMP-1) with data from the validation set. When the additive model from the training set was applied to the data from the validation set, a significant association was showed at $p<0.0001$. This validates the results from the training set - there is an increased risk of subsequent malignancies if subjects have elevated levels of CEA, CA19-9 and TIMP-1 (Fig. 2C and D).

Rank correlations between age and the markers showed that TIMP-1 was associated with age $(\mathrm{r}=0.36, p<0.0001)$ as well as YKL-40 $(\mathrm{r}=0.65, p<0.0001)$ whereas CEA and CA19-9 demonstrated low associations $(\mathrm{r}=0.02, p=0.13$ and $\mathrm{r}=0.01, p=0.72$, respectively).

The analyses of interactions between the different covariates in the training set showed that TIMP-1 was significantly dependent on age for the models with biomarkers on a continuous scale as well as for the dichotomized models. These results demonstrated that elevated TIMP- 1 in younger individuals (50 years) provided a greater risk of malignancy, whereas elevated levels of TIMP-1 in older individuals (70 years) did not show an increased risk. Applying the locked models based on the training set to the validation set confirmed these findings. These hypotheses were not initially specified and therefore interpreted with caution although it was also shown in the validation set. Other cancer-associated biomarkers, such as CA-125 [45-48], prostate specific antigen (PSA) [49-51], different biomarkers related to breast cancer [52, 53] and various DNA-methylations on different genes [54], have been shown to correlate with age biomarkers. 
Inclusion of additional cancer-associated biomarkers may add to the efficacy of identifying subjects at increased risk of developing a subsequent primary malignancy. Additional biomarkers, such as other protein biomarkers, miRNA, ctDNA methylations and DNA fragments [55-65], could be considered. Future combinations of various cancer-associated biomarkers may lead to development of cancer specific risk stratifications based on algorithms, where numeral specific biomarkers and age are included. Age as a covariate should be included in the algorithms as greater age itself leads to an increased risk of subsequent malignancy [66]. Specific calculated malignancy risks may aid in targeting cancer search in individuals with unspecific signs of malignancy and refer these individuals to special cancer investigation departments and/or help specialists at such departments making the right diagnosis [67-71]. In Denmark 'Diagnostic Departments' were established in April 2012. Since then, subjects with unspecified symptoms of severe illness have been referred by their physicians to examination at these departments. Those patients are entitled to an accelerated medical investigation of the symptoms. A pan-cancer biomarker panel could potentially be a part of the examination in such cases, both in Denmark and globally.

\section{Conclusion}

The present study based on pooled data from a population with symptoms attributable to CRC but with non-malignant findings at endoscopy, showed that elevated levels of the cancer-associated biomarkers CEA and CA19-9 were significant in detecting subjects at risk of developing a subsequent primary malignancy. The results were validated in a comparable large population. Furthermore, calculations of interactions between the different covariates showed that the biomarker TIMP-1 was dependent on age in both the training- and the validation set. These results should be validated in other studies, and preferably also in a non-Danish population.

\section{Acknowledgments}

Jorgensen LN (Department of Surgical Gastroenterology, Bispebjerg Hospital, Copenhagen, Denmark), Olsen J (Department of Surgical Gastroenterology, Herlev Hospital, Herlev, Denmark), Rahr HB (Head of Surgery, Vejle Sygehus, Vejle, Denmark), Nielsen KT (Head of Surgery, Randers Hospital, Randers, Denmark), Hoyer U (Head of Mamma Surgery, Aalborg University Hospital, Aalborg, Denmark), Laurberg S (Aarhus University Hospital, Aarhus, Denmark), Brünner N (Department of Veterinary Disease Biology, Faculty of Health and Medical Sciences, University of Copenhagen, Denmark) and the Danish Endoscopy Study Group on Colorectal Cancer Detection are thanked for their contribution and collaboration on the work with the training set.

Jorgensen LN (Department of Surgical Gastroenterology, Bispebjerg Hospital, Copenhagen, Denmark), Madsen MR (Department of Surgical Gastroenterology, Herning Hospital, Herning, Denmark), Vilandt J (Department of Surgical Gastroenterology, Hillerød Hospital, Hillerød, Denmark), Hillig T (Department of Clinical Biochemistry, Hillerød Hospital, Denmark), Klaerke M (Department of Surgical Gastroenterology, Horsens Hospital, Horsens, Denmark), Nielsen KT (Head of Surgery, Randers Hospital, Randers, Denmark), Laurberg S (Aarhus University Hospital, Aarhus, Denmark), Brünner N (Department of Veterinary Disease Biology, Faculty of Health and Medical Sciences, University of Copenhagen, Denmark) and the Danish Endoscopy Study Group on Colorectal Cancer Detection are thanked for their contribution and collaboration on the work with the validation set.

The research nurses, secretaries and technicians at the participating hospital departments and laboratories are thanked for their skillful work. 


\section{Author contributions}

CONCEPTION: Hans J. Nielsen

DATA CURATION: Thomas B. Piper and Ib J. Christensen

ANALYSIS OF DATA: Ib J. Christensen

PREPARATION OF THE MANUSCRIPT: Thomas B. Piper, Hans J. Nielsen and Ib J. Christensen

REVISION FOR IMPORTANT INTELLECTUAL CONTENT: Hans J. Nielsen and Ib J. Christensen

SUPERVISION: Hans J. Nielsen and Ib J. Christensen

\section{Conflict of interest}

Nothing to declare.

\section{Ethical considerations}

The study was approved by the Ethics Committee of the Capital Region of Denmark (KF01-080/03), which represents all collaborating hospitals, and in addition approved by the Danish Data Protection Agency (2003-41-3312). Before inclusion, all subjects signed an informed consent form.

\section{Funding}

The training study received financial support from The Danish Cancer Society, The Kornerup Fund, The Aage and Johanne Louis-Hansen Fund, The Aase and Ejnar Danielsen Fund, The Walter and O. Kristiane Christensen Fund, The Kathrine and Vigo Skovgaard Fund, The 'Midtjyske Bladfund', The Agnes and Poul Friis Fund, The Glunz and Jensen Fund, The Sophus and Astrid Jacobsen Fund, The Arvid Nilsson Fund, The Danish Bank Fund, The Johannes Fog Fund, The Eva and Henry Fraenkel Fund, The Hartmann Bros. Fund, The KID Fund, The Henrik Henriksen Fund, The King Christian X's Fund, The Oda and Hans Svenningsen Fund, The Else and Mogens Wedell-Wedellsborg Fund, The Einar Willumsen Fund, The Willy and Ingeborg Reinhard Fund, The Friedrich and Else Boehm Fund, The Toyota Fund, The IMK Fund, The Danish Medical Research Fund, The Beckett Fund and Hvidovre University Hospital.

The validation study received financial support from The Andersen Isted Fund, The Augustinus Foundation, The Beckett Fund, The Inger Bonnén Fund, The Hans \& Nora Buchard Fund, CEO Jens Bærentsen (private donation, The Walter Christensen Family Fund, The P.M. Christiansen Family Fund, The Aase \& Ejnar Danielsen Fund, The Erichsen Family Fund, The Knud \& Edith Eriksen Fund, The Svend Espersen Fund, The Elna and Jørgen Fagerholt Fund, The Sofus Carl Emil Friis Fund, The Torben \& Alice Frimodt Fund, The Eva \& Henry Frænkel Fund, The Gangsted Fund, The Thora \& Viggo Grove Fund, The H Foundation, The Erna Hamilton Fund, The Sven \& Ina Hansen Fund, The Søren \& Helene Hempel Fund, The Henrik Henriksen Fund, The Jørgen Holm Family Fund, Foundation Jochum, The KID Fund, The Kornerup Fund, The Linex Fund, The Dagmar Marshall Fund, The 'Midtjyske Bladfund', The Axel Muusfeldt Fund, The Børge Nielsen Family Fund, The Michael Hermann Nielsen Fund, The Arvid Nilsson Fund, The Obel Family Fund, The Krista \& Viggo Petersen Fund, The Willy \& Ingeborg Reinhard Fund, The Kathrine \& Vigo Skovgaard Fund, The Toyota Fund, The Vissing Fund, The Wedell-Wedellsborg Fund, Hvidovre University Hospital (The Capital Region of Denmark). 


\section{References}

[1] http://globocan.iarc.fr/Pages/fact_sheets_cancer.aspx.

[2] Fitzmaurice C, et al. Global, Regional, and National Cancer Incidence, Mortality, Years of Life Lost, Years Lived With Disability, and Disability- Adjusted Life-years for 32 Cancer Groups, 1990 to 2015 A Systematic Analysis for the Global Burden of Disease Study. JAMA Oncol. 2017;3(4).

[3] Release P. (2018). www.iarc.who.int/featured-news/latest-global-cancer-data-cancer-burden-rises-to-18-1-millionnew-cases-and-9-6-million-cancer-deaths-in-2018

[4] Brenner H, Kloor M, Pox CP. Colorectal cancer. Lancet. 2014;383(9927):1490-502.

[5] Nielsen HJ, Brnner N, Jorgensen LN, Olsen J, Rahr HB, Thygesen K, et al. Plasma TIMP-1 and CEA in detection of primary colorectal cancer: A prospective, population based study of 4509 high-risk individuals. Scand J Gastroenterol. 2011;46(1):60-9.

[6] Wild CP. (2014). World Cancer Report 2014. Who/Iarc. https://publications.iarc.fr/Non-Series-Publications/WorldCancer-Reports/World-Cancer-Report-2014

[7] Parmeshwar R, Rajan SS, Shrestha K. Principles of cancer screening. Surg (United Kingdom). 2018;36(3):139-44.

[8] Kring TS, Piper TB, Jørgensen LN, Olsen J, Rahr HB, Nielsen KT, et al. Blood-based Biomarkers at Large Bowel Endoscopy and Prediction of Future Malignancies. Biomark Cancer. 2015;7:57-61.

[9] Hvolris MH, Piper TB, Hammer E, Jørgensen LN, Olsen J, Rahr HB, et al. Increased serological cancer-associated biomarker levels at large bowel endoscopy and risk of subsequent primary cancer ${ }^{\dagger}$. Scand $\mathbf{J}$ Gastroenterol. 2016;51(7):860-5.

[10] Piper TB, Jørgensen LN, Olsen J, Nielsen KT, Davis G, Johansen JS, et al. Increased serological, cancer-associated protein biomarker levels at diagnosis of large bowel adenoma: Risk of subsequent primary malignancy? Acta Oncologica. 2018;58(sup 1: Advanced Adenomas of the Colon and Rectum), 42-8.

[11] Bonnema DD, Webb CS, Pennington WR, Stroud RE, Leonardi AE, Clark LL, McClure CD, Finklea L, Spinale FG ZM. Effects of age on plasma matrix metalloproteinases (MMPs) and tissue inhibitor of metalloproteinases (TIMPs). J Card Fail. 2007;13(7):530-40.

[12] Sørensen J, Subhi Y, Molbech CR, Nielsen MK, Sørensen TL. Plasma levels of matrix metalloprotease MMP-9 and tissue inhibitor TIMP-1 in caucasian patients with polypoidal choroidal vasculopathy. Vision (Switzerland). 2020;4(27).

[13] Holten-Andersen MN, Murphy G, Nelsen HJ, Pedersen AN, Christensen IJ, Høyer-Hansen G, et al. Quantitation of TIMP-1 in plasma of healthy blood donors and patients with advanced cancer. Br J Cancer. 1999;80(3-4):495-503.

[14] Cancer C, Holten-andersen MN, Christensen IJ, Nielsen HJ, Stephens RW, Jensen V, et al. Total Levels of Tissue Inhibitor of Metalloproteinases 1 in Plasma Yield High Diagnostic Sensitivity and Specificity in Patients with Colon Cancer. Clinical Cancer Research. 2002;8(January 2002):156-64.

[15] Cintin C, Johansen JS, Christensen IJ. PA Price SS and HN. Serum YKL-40 and colorectal cancer. Br J Cancer. 1999;79:1494-9.

[16] Cintin C, Johansen JS, Christensen IJ, Price PA, Sørensen S, Nielsen HJ. High serum YKL-40 level after surgery for colorectal carcinoma is related to short survival. Cancer. 2002;95(2):267-74.

[17] Behbehani AI, Al-Sayer H, Farghaly M, Kanawati N, Mathew A, Al-Bader A, et al. Prognostic significance of CEA and CA 19-9 in colorectal cancer in Kuwait. Int J Biol Markers. 2000;15(1):51-5.

[18] Hundt S, Haug U, Brenner H. Blood Markers for Early Detection of Colorectal Cancer: A Systematic Review. Cancer Epidemiol Biomarkers Prev. 2007;16(10):1935-53.

[19] Holten-Andersen MN, Murphy G, Nielsen HJ, Pedersen AN, Christensen IJ, et al. Quantitation of TIMP-1 in plasma of healthy blood donors and patients with. British Journal of Cancer. 1999;80(3/4):495-503.

[20] Møller Sørensen N, Vejgaard Sørensen I, Ørnbjerg Würtz S, Schrohl A-S, Dowell B, Davis G, et al. Biology and potential clinical implications of tissue inhibitor of metalloproteinases-1 in colorectal cancer treatment. Scand J Gastroenterol. 2008;43(7):774-86.

[21] Johansen JS, Christensen IJ, Jorgensen LN, Olsen J, Rahr HB, Nielsen KT, et al. Serum YKL-40 in Risk Assessment for Colorectal Cancer: A Prospective Study of 4,496 Subjects at Risk of Colorectal Cancer. Cancer Epidemiol Biomarkers Prev. 2015;24(3):621-6.

[22] Allin KH, Bojesen SE, Johansen JS, Nordestgaard BG. Cancer risk by combined levels of YKL-40 and C-reactive protein in the general population. Br J Cancer. 2012;106(1):199-205.

[23] Kjaergaard AD, Nordestgaard BG, Johansen JS, Bojesen SE. Observational and genetic plasma YKL-40 and cancer in 96,099 individuals from the general population. Int J Cancer. 2015;137(11):2696-704.

[24] Waas ET, Hendriks T, Lomme RM, W. T. Plasma levels of matrix metalloproteinase-2 and tissue inhibitor of metalloproteinase-1 correlate with disease stage and survival in colorectal cancer patients. Diseases of the Colon \& Rectum. 2005;48(4):700-10. 
[25] Weigang W, Xiaoqin X, Baoguo T, Yan W, Lili D, et al. The diagnostic value of serum tumor markers CEA, CA19-9, CA125, CA15-3, and TPS in metastatic breast cancer. Clinica Chimica Acta. 2017;470:51-5.

[26] Lertkhachonsuk A aroon, Buranawongtrakoon S, Lekskul N, Rermluk N, Wee-Stekly WW, Charakorn C. Serum CA199, CA-125 and CEA as tumor markers for mucinous ovarian tumors. J Obstet Gynaecol Res. 2020;46(11):2287-91.

[27] Liu X, Meng X, Li Y, Chai W, Qian J, Tang H. Role of CA19-9 in the prognostic evaluation of SOX neoadjuvant chemotherapy for gastric cancer. 2018;11(11):5387-93.

[28] Van Der Kaaij RT, Voncken FEM, Van Dieren JM, Snaebjornsson P, Korse CM, Grootscholten C, et al. Elevated pretreatment CEA and CA19-9 levels are related to early treatment failure in esophageal adenocarcinoma. Am J Clin Oncol Cancer Clin Trials. 2019;42(4):345-50.

[29] Hsu CC, Goyal A, Iuga A, Krishnamoorthy S, Lee V, Verna EC, et al. Elevated CA19-9 is Associated with Increased Mortality in A Prospective Cohort of Hepatocellular Carcinoma Patients. Clin Transl Gastroenterol. 2015;6(2):e74-9.

[30] Pesta M, Kucera R, Topolcan O, Karlikova M, Houfkova K, Polivka J, et al. Plasma microRNA levels combined with CEA and CA19-9 in the follow-up of colorectal cancer patients. Cancers (Basel). 2019;11(6):864.

[31] Aljada IS, Ramnath N, Donohue K, Harvey S, Brooks JJ, Wiseman SM, et al. Upregulation of the tissue inhibitor of metalloproteinase-1 protein is associated with progression of human non-small-cell lung cancer. J Clin Oncol. 2004;22(16):3218-29.

[32] Cho NH, Shim HS, Rha SY, Kang SH, Hong SH, Choi YD, et al. Increased Expression of Matrix Metalloproteinase 9 Correlates with Poor Prognostic Variables in Renal Cell Carcinoma. Eur Urol. 2003;44(5):560-6.

[33] Manenti L, Paganoni P, Floriani I, Landoni F, Torri V, Buda A, Taraboletti G, Labianca R, Belotti D GR. Expression levels of vascular endothelial growth factor, matrix metalloproteinases 2 and 9 and tissue inhibitor of metalloproteinases 1 and 2 in the plasma of patients with ovarian carcinoma. Eur J Cancer. 2003;39(13):1948-56.

[34] Johansen JS, Jensen BV, Roslind A, Nielsen D, Price PA. Serum YKL-40, a new prognostic biomarker in cancer patients? Cancer Epidemiol Biomarkers Prev. 2006;15(2):194-202.

[35] Liu MC, Oxnard GR, Klein EA, Swanton C, Seiden MV, Liu MC, et al. Sensitive and specific multi-cancer detection and localization using methylation signatures in cell-free DNA. Ann Oncol. 2020;31(6):745-59.

[36] Lennon AM, Buchanan AH, Kinde I, Warren A, Sheridan K, Rosica D, et al. Feasibility of blood testing combined with PET-CT to screen for cancer and guide intervention. Science (80-). 2020;369(6499).

[37] Wilhelmsen M, Christensen IJ, Rasmussen L, Jørgensen LN, Madsen MR, Vilandt J, et al. Detection of colorectal neoplasia: Combination of eight blood-based, cancer-associated protein biomarkers. Int J Cancer. 2017;140(6):1436-46.

[38] Gray JPF \& RJ. A Proportional Hazards Model for the Subdistribution of a Competing Risk. J Am Stat Assoc. 1999;94(446):496-509.

[39] https://www-dep.iarc.fr/NORDCAN/DK/frame.asp

[40] KRÆFTPROFIL - Prostatakræft 2000-2007. (2009). https://www.sst.dk/ /media/CB3BFDCA42C4463AA40C02BE6 1A98E46.ashx

[41] Outzen M, Brasso K, Martinussen N, Christensen J, Friis S, Olsen A, et al. Prostate cancer in Denmark 1978 - 2009 trends in incidence and mortality. Acta Oncol (Madr). 2013;52:831-6.

[42] https://www.esundhed.dk/Registre/Cancerregisteret/Nye-kraefttilfaelde.

[43] https://www.cancer.gov/about-cancer/causes-prevention/risk/age.

[44] White MC, Holman DM, Boehm JE, Peipins LA, Browning R. Age and Cancer Risk A Potentially Modifiable Relationship. Am J Prev Med. 2014;46(3):S7-15.

[45] Pauler DK, Menon U, Mcintosh M, Symecko HL, Skates SJ, Jacobs IJ. Factors Influencing Serum CA125II Levels in Healthy Postmenopausal Women. 2001;10(5):489-93.

[46] Sasamoto N, Babic A, Rosner BA, Fortner RT, Vitonis AF, Yamamoto H, et al. Development and validation of circulating CA125 prediction models in postmenopausal women. J Ovarian Res. 2019;4:1-12.

[47] Fortner RT, Vitonis AF, Schock H, Hüsing A, Johnson T, Fichorova RN, et al. Correlates of circulating ovarian cancer early detection markers and their contribution to discrimination of early detection models: results from the EPIC cohort. J Ovarian Res. 2017;10(1):20.

[48] Johnson CC, Kessel B, Riley TL, Ragard LR, Williams CR, Xu JL, et al. The epidemiology of CA-125 in women without evidence of ovarian cancer in the Prostate, Lung, Colorectal and Ovarian Cancer (PLCO) Screening Trial. Gynecol Oncol. 2008;110(3):383-9.

[49] Oesterling JE. Age-Specific Reference Ranges for Serum PSA. N Engl J Med. 1996;335(5):345-6.

[50] Bosch JLHR, Tilling K, Bohnen AM, Donovan JL. Establishing Normal Reference Ranges for PSA Change With Age in a Population-Based Study: The Krimpen Study. The Prostate. 2006;66(4):335-43.

[51] Battikhi MNG. Age-specific reference ranges for prostate-specific antigen (PSA) in Jordanian patients. Nat Publ Gr. 2003;256-60. 
[52] Morrison DH, Rahardja D, King E, Peng Y, Sarode VR. Tumour biomarker expression relative to age and molecular subtypes of invasive breast cancer. Br J Cancer. 2012;107(2):382-7.

[53] Mariani A, Sebo TJ, Katzmann JA, Riehle DL, Dowdy SC, Keeney GL, et al. HER-2 / neu Overexpression and Hormone Dependency in Endometrial Cancer: Analysis of Cohort and Review of Literature *. Anticancer Res. 2005;2928:2921-7.

[54] Wang Y, Zhang J, Xiao X, Liu H, Wang F, Li S, et al. The identification of age-associated cancer markers by an integrative analysis of dynamic DNA methylation changes. Scientific Reports. (2016);6(22722).

[55] Holdenrieder S, Dharuman Y, Standop J, Trimpop N, Herzog M, Hettwer K, Simon K, Uhlig S, Micallef J. Novel serum nucleosomics biomarkers for the detection of colorectal cancer. Anticancer Research. 2014;34(5):2357-62.

[56] Phallen J, Sausen M, Adleff V, Leal A, Hruban C, White J, et al. Direct detection of early-stage cancers using circulating tumor DNA. 2017;2415(August).

[57] Rasmussen L, Wilhelmsen M, Christensen IJ, Andersen J, Nannestad L. Protocol Outlines for Parts 1 and 2 of the Prospective Endoscopy III Study for the Early Detection of Colorectal Cancer: Validation of a Concept Based on Blood Biomarkers Corresponding Author: JMIR Res Protoc. 2016;5(3).

[58] Schøler LV, Reinert T, Mai-britt W, Kassentoft CG, Vang S, Nordentoft I, et al. Clinical Implications of Monitoring Circulating Tumor DNA in Patients with Colorectal Cancer. 2017;5437-46.

[59] Y Nakamura TY. Clinical Utility of Analyzing Circulating Tumor DNA in Patients with Metastatic Colorectal Cancer. Oncologist. 2018;23:1310-8.

[60] Routman DM, Chera BS, Gaorav P. Circulating Tumor DNA Biomarkers for Early Detection of Oligometastasis. 2020;116-23.

[61] Thorsen SF, Gromova I, Christensen IJ, Fredriksson S. Gel-Based Proteomics of Clinical Samples Identifies Potential Serological Biomarkers for Early Detection of Colorectal Cancer. Int J Mol Sci. 2019;20(6082):1-19.

[62] Cristiano S, Leal A, Phallen J, Fiksel J, Bruhm DC, Jensen S $\varnothing$, et al. Genome-wide cell-free DNA fragmentation in patients with cancer. Nature. 2019;570(7761):385-9.

[63] Jensen S $\varnothing, \varnothing$ gaard N, Ørntoft MW, Rasmussen MH, Bramsen JB, Kristensen H, et al. Novel DNA methylation biomarkers show high sensitivity and specificity for blood- based detection of colorectal cancer - a clinical biomarker discovery and validation study. Clin Epigenetics. 2019;11(158):1-14.

[64] Janhavi R, Raut ZG, Petra Schrotz-King HB. Whole-blood DNA Methylation Markers for Risk Stratification in Colorectal Cancer Screening: A Systematic Review. Cancers (Basel). 2019;11(912):1-23.

[65] Hariharan R, Jenkins M. Utility of the methylated SEPT9 test for the early detection of colorectal cancer: a systematic review and meta-analysis of diagnostic test accuracy. BMJ Open Gastro. 2020;9:1-8.

[66] Pilleron S, Sarfati D, Janssen-heijnen M, Vignat J, Ferlay J, et al. Global cancer incidence in older adults, 2012 and 2035: A population-based study. Int J Cancer. 2019;144(1):49-58.

[67] Cohen JD, Li L, Wang Y, Thoburn C, Afsari B, Danilova L, et al. Detection and localization of surgically resectable cancers with a multi-analyte blood test. Science. 2018;359(6378):926-30.

[68] Wilhelmsen M, Christensen IJ, Jørgensen LN, Madsen MR, Vilandt J, et al. Clean Colorectum at Diagnostic Colonoscopy: Subsequent Detection of Extracolonic Malignancies by Plasma Protein Biomarkers? Biomarkers in Cancer. 2018;10:1-9.

[69] Prades J, Espinás JA, Font R, Argimon JM, Borrs JM. Implementing a Cancer Fast-track Programme between primary and specialised care in Catalonia (Spain): A mixed methods study. Br J Cancer. 2011;105(6):753-9.

[70] Jørgensen SF, Ravn P, Thorsen S, Worm SW. Characteristics and outcome in patients with non-specific symptoms and signs of cancer referred to a fast track cancer patient pathway; a retrospective cohort study. BMC Cancer. 2017;17(1):111.

[71] Jensen H, Tørring ML, Vedsted P. Prognostic consequences of implementing cancer patient pathways in Denmark: A comparative cohort study of symptomatic cancer patients in primary care. BMC Cancer. 2017;17(1):1-10. 